\title{
Longitudinal effects of antibiotics and fecal transplant on lemur gut microbiota structure, associations, and resistomes
}

Sally L. Bornbusch ${ }^{1 \dagger}$, Rachel L. Harris ${ }^{1}$, Nicholas M. Grebe ${ }^{1}$, Kimberly Roche $^{2}$, Kristin DimacStohl ${ }^{1}$, Christine M. Drea ${ }^{1}$

${ }^{1}$ Department of Evolutionary Anthropology, Duke University

${ }^{2}$ Program in Computational Biology \& Bioinformatics, Duke University

$\dagger$ - corresponding author: sally.bornbusch@gmail.com

\section{Abstract:}

Antibiotics alter the diversity, structure, and dynamics of host-associated microbial consortia, including via development of antibiotic resistance; however, patterns of recovery from dysbiosis

17 and methods to mitigate negative effects, remain poorly understood. We applied an ecological

18 framework via long-term, integrated study of community structure, across scales, to improve

19 understanding of host-microbe symbiosis during dysbiosis and recovery. We experimentally

20 administered a broad-spectrum antibiotic alone or with subsequent fecal transfaunation to

21 healthy, male ring-tailed lemurs (Lemur catta) and longitudinally tracked the diversity,

22 composition, associations, and resistomes of their gut microbiota. Whereas microbial diversity

23 recovered rapidly in lemurs, antibiotics caused long-term instability in community composition -

24 effects that were attenuated by fecal transfaunation. Antibiotic resistance genes, which were

25 universally present, including in treatment-naïve subjects, increased during and persisted after

26 antibiotic treatment. Long-term, integrated study post antibiotic-induced dysbiosis revealed

27 differential, metric-dependent evidence of recovery, beneficial effects of fecal transfaunation, and negative consequences to lemur resistomes. 


\section{Introduction}

32 The long, co-evolutionary history between vertebrates and their microbes underpins the

33 complex web of interactions linking commensal microbiota to host function ${ }^{1,2}$. Because

34 perturbations to these communities can have both short- and long-term, negative consequences ${ }^{3-}$

35 , we increasingly recognize the benefits provided by our endogenous microbiota and have come

36 to view them as 'old friends' ${ }^{6,7}$. To exemplify, while antibiotic treatment effectively combats

37 immediate bacterial infections, it can also lead to prolonged, severe negative side-effects, such as

38 the elimination of beneficial microbes and the deterioration of microbiome function ${ }^{8,9}$.

39 Moreover, antibiotics also promote changes in microbial genomes; the ubiquitous use of

40 antibiotics has spurred the spread of genes encoding antibiotic resistance (ABR), which can have

41 potentially catastrophic consequences ${ }^{10}$. Microbial therapies, such as fecal transfaunation, can

42 mitigate the detrimental side-effects of antibiotics ${ }^{11}$; however, because antibiotics are often

43 studied in the context of preexisting illness or injury (which independently influences microbial

44 communities), the severity, duration, and recovery from dysbiosis owing purely to antibiotics

45 remain unclear. Here, we apply an ecological framework in healthy animals to better understand

46 the trajectory and processes governing recovery or return to a stable microbial community

47 following antibiotic-induced dysbiosis. Because nonhuman primates are prime models in which

48 to probe microbial dynamics and the development of ABR in response to antibiotic treatment, we

49 experimentally administered a broad-spectrum antibiotic to male ring-tailed lemurs (Lemur

50 catta) and used a longitudinal approach to track impacts on the composition and resistomes of

51 their gut microbiota. We further tested the effects of fecal transfaunation as an intervention to

52 promote the recovery of microbial composition and to potentially mitigate the development and

53 persistence of $\mathrm{ABR}$. 
Antibiotics and ABR genes have ancient origins as natural compounds or genetic defenses,

55 respectively, used by microbes to compete and survive in densely populated communities,

56 whether within or outside of a host ${ }^{12,13}$. The ability of bacteria to rapidly undergo mutation ${ }^{14,15}$

57 and share advantageous genes via lateral gene transfer ${ }^{16,17}$ has resulted in myriad, naturally

58 occurring ABR genes ${ }^{18,19}$. The response of a microbial community to natural antibiotics is

59 largely dictated by the interactions between microbial taxa, which vary over time and across

60 environments. The efficacy and ubiquity of man-made antibiotics have severely perturbed

61 microbial communities via targeted (e.g. narrow spectrum) or indiscriminate (e.g. broad

62 spectrum) elimination of bacterial groups ${ }^{20}$, thereby altering the composition and, ultimately,

63 functional potential of microbiomes ${ }^{20-22}$. In addition, these antibiotics have magnified selective

64 pressure on bacterial communities, making ABR genes advantageous and instigating their

65 proliferation ${ }^{23,24}$, thereby altering the microbiota's genomic make-up. Within host-associated

66 microbiomes, the propagation of ABR can result in virulent, resistant pathogens ${ }^{25,26}$ that reduce

67 the diversity of native or beneficial microbes ${ }^{27,28}$ and diminish immune capacity of the host. Our

68 understanding of these phenomena primarily derives from studies that characterize the effects of

69 antibiotics on the elimination or development of ABR within specific bacterial pathogens ${ }^{29,30}$.

70 We know comparatively less about how man-made antibiotics influence the aggregate

71 interactions within presumed healthy, host-associated communities and how those dynamics

72 influence the recovery of microbiota.

73 Recognizing that commensal consortia are vital to the host has spurred increased research

74 into microbial therapies to mitigate the negative consequences of dysbiosis. In fecal

75 transfaunation, for example, a 'healthy' or 'native' community of microbes sourced from feces is

76 transferred into a dysbiotic community to combat pathogens and promote the growth of 
77 beneficial microbes ${ }^{31,32}$. Because coprophagy (the ingestion of fecal material either directly or

78 via prey consumption) bolsters gut microbiota during development or illness ${ }^{33,34}$, medical

79 practitioners have examined the use of fecal transfaunations to treat gastrointestinal distress in a

80 wide range of host taxa $\mathrm{a}^{35,36}$. As in studies of antibiotics, however, the effects of fecal

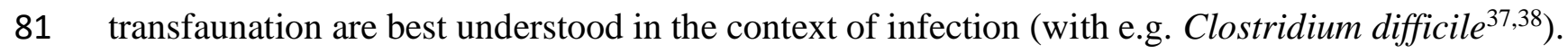

82 Whether or not fecal transfaunation alters the trajectory of microbiome recovery more broadly

83 remains unclear.

84 Understudied compared to anthropoid primates, lemurs have a unique evolutionary trajectory

85 that makes them interesting models in which to study the dynamics between hosts and their co-

86 evolved microbes ${ }^{39-42}$. Ring-tailed lemurs are ecologically flexible ${ }^{43,44}$, owing in part to their

87 highly omnivorous diet, making them one of the few lemur species to thrive in captivity. This

88 flexibility is reflected in their resilient gut microbiota that seem relatively unperturbed by aspects

89 of captivity ${ }^{42}$. Ring-tailed lemurs re also relatively robust to health concerns, such as

90 gastrointestinal problems, that affect the microbiota and welfare of other captive strepsirrhines ${ }^{45}$.

91 Here, we apply classic ecological principles to gut microbial communities to investigate two

92 non-exclusive hypotheses regarding post-dysbiosis recovery. We use experimental manipulations

93 (antibiotic treatment with or without fecal transfaunation), paired with longitudinal data to

94 examine patterns in microbiota structure (e.g. alpha and beta diversity via 16S rRNA amplicon

95 sequencing), bacterial associations (via Bayesian models of covariation), and ABR gene profiles

96 (via shotgun metagenomic sequencing). First, diversity increases functional redundancy within a

97 community and thus improves stability ${ }^{46-48}$. Under the 'diversity begets stability' hypothesis, as

98 applied to a dysbiotic microbiome, recovery of alpha diversity, regardless of microbial identity,

99 should be vital and sufficient to achieve a stable microbiome ${ }^{49-51}$. Accordingly, after antibiotic 
treatment, we would expect to see an increase in microbial richness (e.g. alpha diversity), independent of fecal transfaunation. The resulting stable communities of the two treatment

102 groups could thus have similar richness, but different compositions. Alternately, previous

103 evidence also indicates that certain community members (i.e., keystone species or specific 'old

104 friends') are foundational to community function ${ }^{52-55}$, such that recovery of a stable microbiome

105 requires specific community composition (e.g. beta diversity). Under the 'key-stone species'

106 hypothesis, we would predict that, following antibiotic-mediated dysbiosis, there would be

107 recovery of the same community composition, with fecal transfaunation accelerating the

108 recovery rate. Accordingly, the resulting stable communities of both treatment groups would

109 have similar compositions. These two hypotheses could also work in concert, but along different

110 schedules, with potentially more rapid recovery of richness, but slower and more variable

111 recovery of composition. Notably, the complexity of dynamics between specific community

112 members (i.e., cooperation and competition) could create long-term fluctuations in community

113 composition that would be highlighted by bacterial covariations between key members of the

114 community. Furthermore, the presence of ABR within the microbiomes could exert a distinct

115 force in driving community composition during the treatment and recovery phases. By tracking

116 ABR prevalence and type, coupled with bacterial covariation, we can make inferences about

117 which microbes may be harboring and expressing ABR genes.

\section{Results}

Baseline and control bacterial communities

121 In the pretreatment phase, neither alpha nor beta diversity varied significantly between the

122 three experimental groups (alpha diversity: Kruskal-Wallis test, $\mathrm{H}=2.478, \mathrm{p}=0.289$, beta 

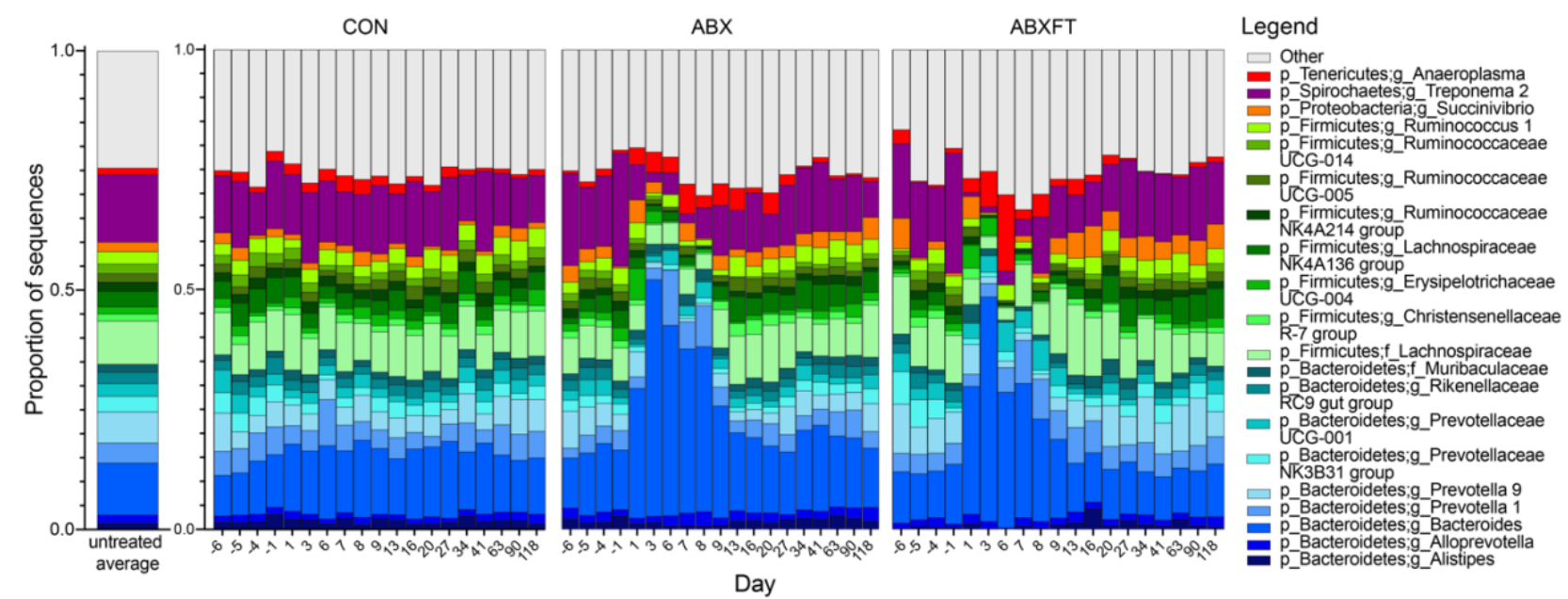

Figure 1. Mean relative abundances of bacterial genera over time in the gut microbiomes of three experimental groups of male ring-tailed lemurs (Lemur catta). Shown are values for healthy animals that received no treatment $(\mathrm{CON})$, antibiotics only $(\mathrm{ABX})$, or antibiotics plus fecal transfaunation (ABXFT). Genera are identified by color; those representing $<1 \%$ of the microbiomes were combined into the category "Other". The $\mathrm{x}$ axis shows day relative to three phases of study: pretreatment (days -6 to -1), treatment (day 0-6/7), and recovery (day7/8-118).

diversity: $\mathrm{H}=2.658, \mathrm{p}=0.264$ ). Across all control samples (across all phases of study; $\mathrm{n}=184$ ),

diversities of CON animals, remained relatively stable over each year's four-month study period, showing consistency across the breeding season. Adding the pretreatment phase of the other two

127 groups (baseline samples; $n=43$ ) to the control group (see 'untreated average' in Figure 1), the

128 bacterial gut microbiota of healthy male ring-tailed lemurs, in captivity, were dominated by taxa

in the Bacteroidetes and Firmicutes phyla, with lesser contributions from Proteobacteria,

Spirochaetes, and Tenericutes. Within these five phyla, 20 genera accounted for minimally $1 \%$ of the total sequences (Figure 1).

Response to and recovery from antibiotic treatment: alpha diversity and microbial membership 
lower scores (HGAM: CON vs. ABX, $\mathrm{t}=-3.535, \mathrm{p}<0.001 ; \mathrm{CON}$ vs. ABXFT, $\mathrm{t}=-4.007, \mathrm{p}<$

year*experimental condition, $\mathrm{F}=0.942, \mathrm{p}=0.391$ ), suggesting consistency of effects across both

years.

As expected for the treatment phase, antibiotic-treated (ABX and ABXFT) animals showed a

142 dramatic reduction in alpha diversity relative to CON animals (day 0-6; HGAM: CON vs. ABX,

$143 \mathrm{t}=-4.534, \mathrm{p}<0.001 ; \mathrm{CON}$ vs. ABXFT, $\mathrm{t}=-3.754, \mathrm{p}<0.001 ;$ see shaded bar in Figure 2).

144 Consistent with the broad effects of amoxicillin, antibiotic treatment in healthy lemurs was

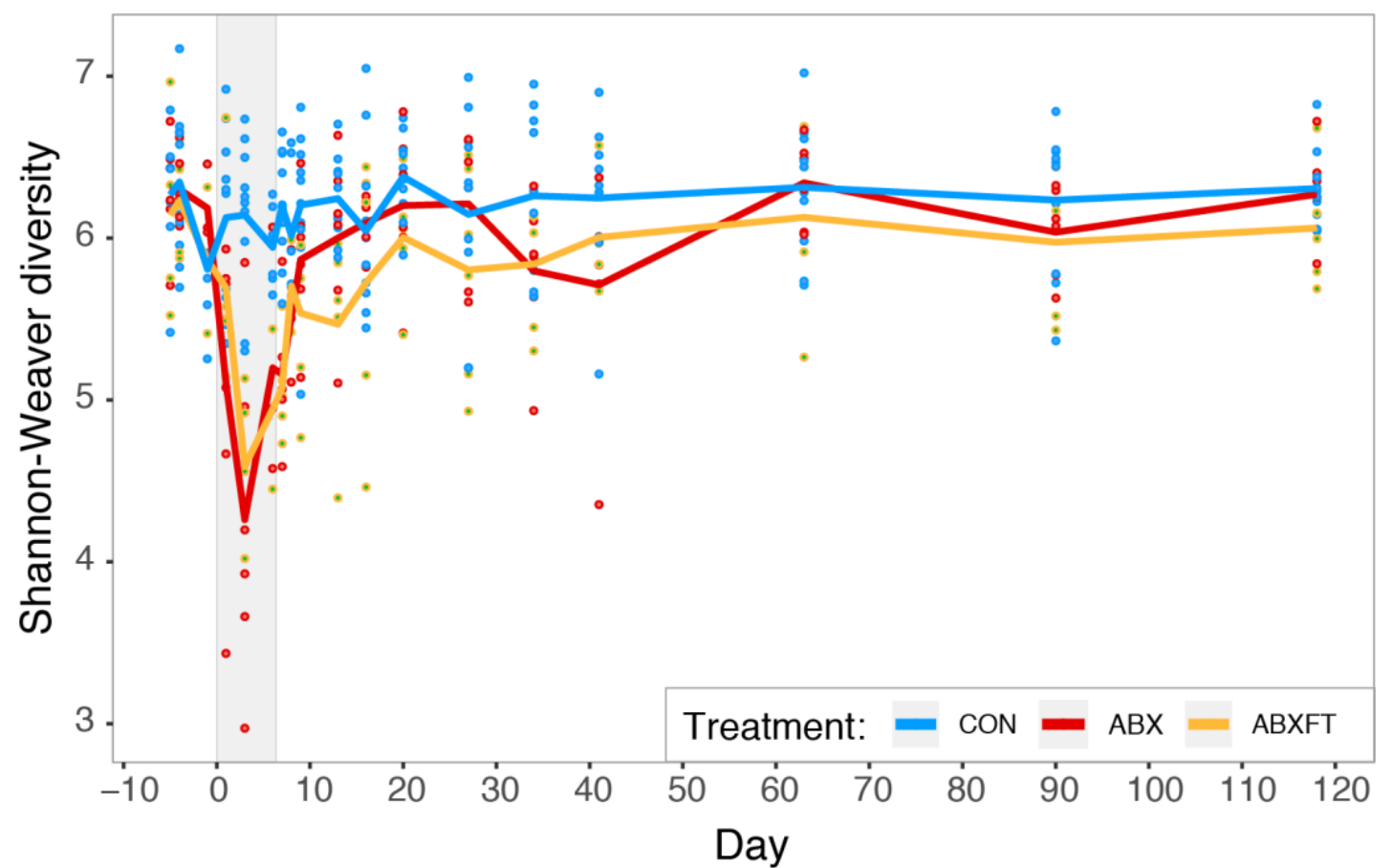

Figure 2. Shannon-Weaver alpha diversity over time in three experimental groups of male ringtailed lemurs (Lemur catta). Shown are values for healthy animals that received no treatment $(\mathrm{CON})$, antibiotics only (ABX), or antibiotics plus fecal transfaunation (ABXFT). Dots represent individual data points and lines connect the mean values of alpha diversity across individuals at each time point. The shaded window represents the period of antibiotic treatment (day 0-6), with fecal transfaunation administered on day 7; all values prior to the onset of treatment represent baseline values and all values post-treatment represent the period of recovery. 
146 numerous taxa in the Firmicutes phylum, such as members of the Clostridiales class (e.g.

147 Ruminococcaceae and Lachnospiraceae families). Certain taxa, however, were markedly

148 unaffected by antibiotic treatment, including the Bacteroides genus and other members of the

149 Bacteroidales family.

150 When focusing on the recovery phase only, we found that the differences in alpha diversity

151 between CON and antibiotic-treated animals persisted over the nearly four-month, post-treatment

152 period, suggesting long-lasting dysbiosis. Compared to CON animals, antibiotic-treated groups

153 maintained significantly lower alpha diversity (HGAM: CON vs. ABX, $t=-2.256, p=0.025$;

154 CON vs. ABXFT, $\mathrm{t}=-3.036, \mathrm{p}=0.002)$; however, there was no significant differences between

155 the alpha diversities of $\mathrm{ABX}$ and ABXFT animals during recovery (HGAM: ABX vs. ABXFT, $t$

$156=0.931, p=0.354$; Figure 2), suggesting no benefits of fecal transfaunation on alpha diversity.

157 Unexpectedly, however, we observed an initial, rapid increase in alpha diversity in both

158 experimental groups, consistent with the 'diversity begets stability' hypothesis (Figure 2).

159

160

Response to and recovery from antibiotic treatment: beta diversity 


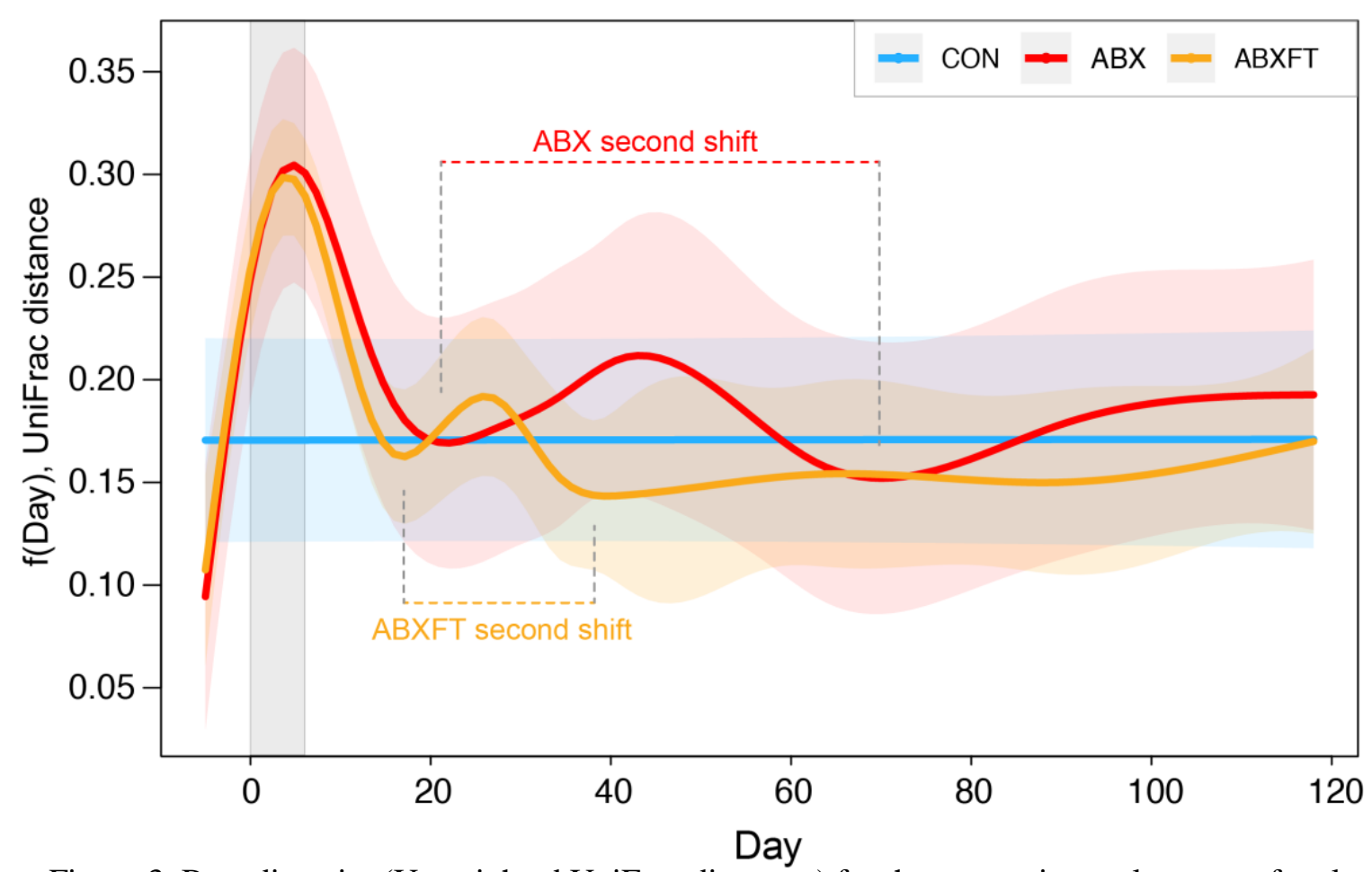

Figure 3. Beta diversity (Unweighted UniFrac distances) for three experimental groups of male ringtailed lemurs (Lemur catta), represented as model-predicted distances from baseline, with 95\% confidence intervals. Shown are values for healthy animals that received no treatment (CON), antibiotics only $(\mathrm{ABX})$, or antibiotics plus fecal transfaunation (ABXFT). The gray shaded window represents the period of antibiotic treatment, with the prior period representing baseline and the subsequent period representing recovery. The second shifts away from baseline are identified and labelled for $\mathrm{ABX}$ and $\mathrm{ABXFT}$ animals.

Across all three phases, we also found experimental condition to be a significant predictor of

162 beta diversity (HGAM2: $\mathrm{F}=5.625, \mathrm{p}=0.004$; Figure 3 ), but in a manner that differed from the

163 findings on alpha diversity. Notably, compared to CON animals, ABX, but not ABXFT animals,

164 showed significantly greater distances from their baseline communities (HGAM: CON vs. ABX,

$165 \mathrm{t}=3.434, \mathrm{p}<0.001 ; \mathrm{CON}$ vs. ABXFT, $\mathrm{t}=1.726, \mathrm{p}=0.085)$. Nevertheless, during the treatment

166 phase, specifically, and compared to CON animals, both groups of antibiotic-treated animals

167 showed significantly greater distances from their baseline communities (HGAM: CON vs. ABX,

$168 \mathrm{t}=-3.847, \mathrm{p}<0.001 ; \mathrm{CON}$ vs. ABXFT, $\mathrm{t}=-3.761, \mathrm{p}<0.001)$. Therefore, the recovery

169 trajectories of the two treated groups diverged post treatment. 
Indeed, during the recovery phase, $\mathrm{CON}$ animals were significantly less distant from baseline

171 when compared to ABX animals, but not when compared to ABXFT animals (HGAM: CON vs.

172 ABX, $\mathrm{t}=2.790, \mathrm{p}=0.005 ; \mathrm{CON}$ vs. ABXFT, $\mathrm{t}=0.599, \mathrm{p}=0.549)$, consistent with the 'key-

173 stone species' hypothesis. Furthermore, when comparing the recoveries of the two antibiotic-

174 treated groups, ABX animals had significantly greater distance from baseline compared to

175 ABXFT animals (HGAM: $t=2.115, p=0.036$; Figure 3 ). The bacterial composition of ABX

176 animals continued to oscillate throughout the course of the experiment, whereas in ABXFT

177 animals, bacterial composition became relatively stable approximately 2 weeks after the

178 treatment phase (Figure 3), further supporting the results reported above. Specifically, after the

179 first compositional shift during the treatment period, the bacterial composition of both $\mathrm{ABX}$ and

180 ABXFT animals underwent a second shift away from baseline during the recovery period;

181 however, the magnitude and span of these secondary shifts differed between the ABX and ABFT

182 groups (Figure 3).

\section{Bacterial associations}

To characterize the bacterial covariations that underlie microbial dynamics in the lemurs'

186 gut, we used pairwise covariation analyses; we detected several strong covariations $(\rho>0.5$ or $\rho$

$187<-0.5$; hereafter 'associations') between pairs of microbes within the lemurs' gut microbiomes

188 (Table S1). We investigated these associations under all three experimental conditions in two

189 stages: across all experimental phases and during the recovery phase.

190 Minimal variation within the microbiota of CON animals limited the detectability of normal

191 bacterial associations; nevertheless, two strong associations emerged. The first was between the

192 genus Cerasicoccus and the order WCHB1-41 and was evident across all experimental phases; 
193 the second was between the genus Cerasicoccus and the order Rhodospirillales, and was evident

194 during the recovery phase (Table S1). These two relationships reflect the small-scale, yet ever-

195 present, microbial dynamics that occur in healthy, unperturbed microbiomes.

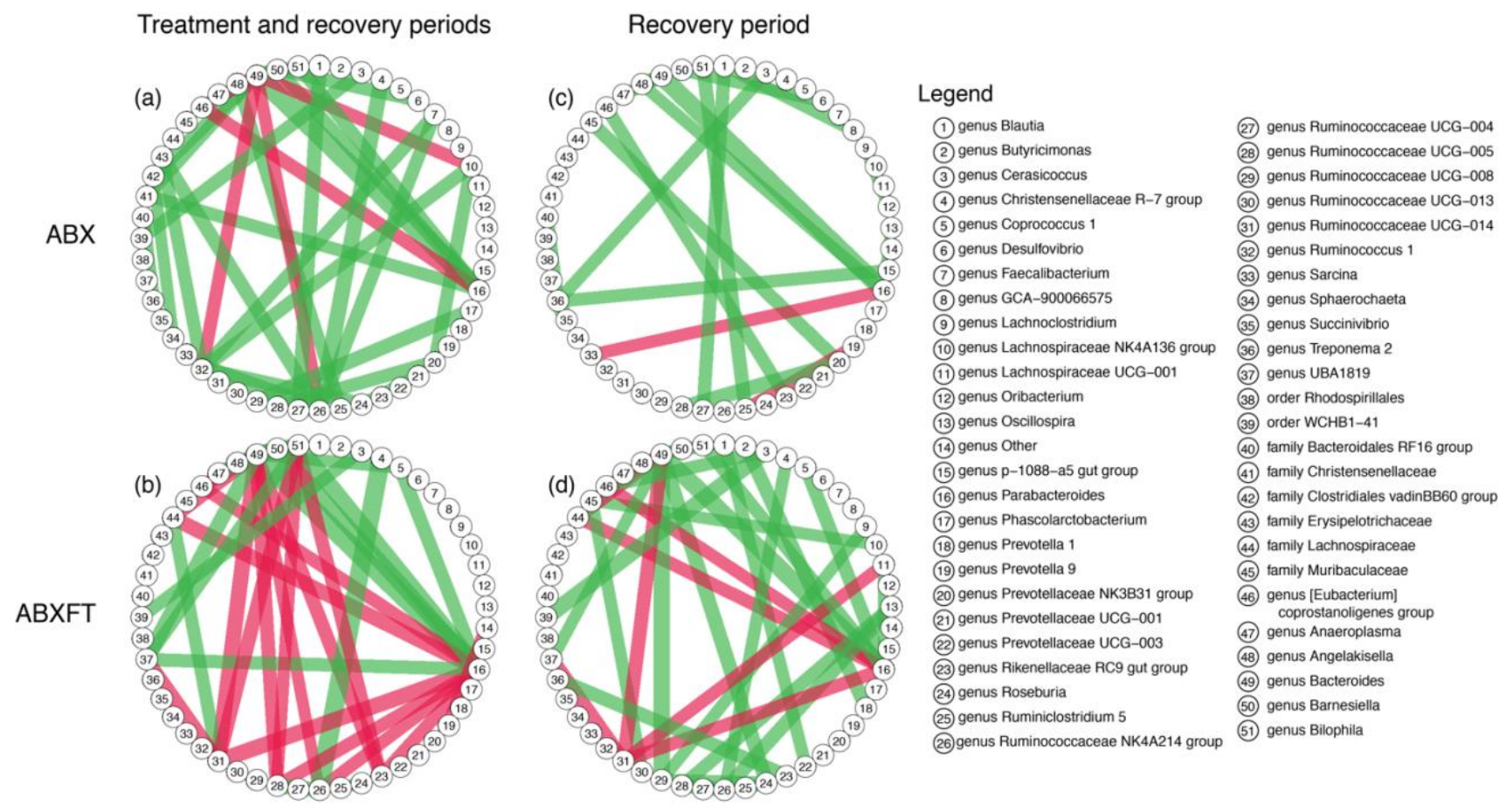

Figure 4. Bacterial associations for healthy, male ring-tailed lemurs (Lemur catta) either treated with antibiotics only (ABX) or with antibiotics plus a fecal transfaunation (ABXFT). Line colors represent the direction of the correlation (green $=$ positive, red $=$ negative); line width is scaled to the magnitude of the correlation.

197 all three experimental phases, there were 35 and 31 strong associations, respectively (Figures

4a,b). In ABX animals, these associations were predominately positive, with only six negative

associations, whereas in ABXFT lemurs, positive and negative associations were equally 
ABXFT animals (Table S1). Moreover, in ABX and ABXFT animals, the log ratios of

Parabacteroides and Bacteroides abundances both showed increases during the treatment phase,

207 majority of strong pairwise associations with Parabacteroides or Bacteroides were positive,

208 indicating that the associated taxa also withstood the effects of antibiotic treatment, potentially
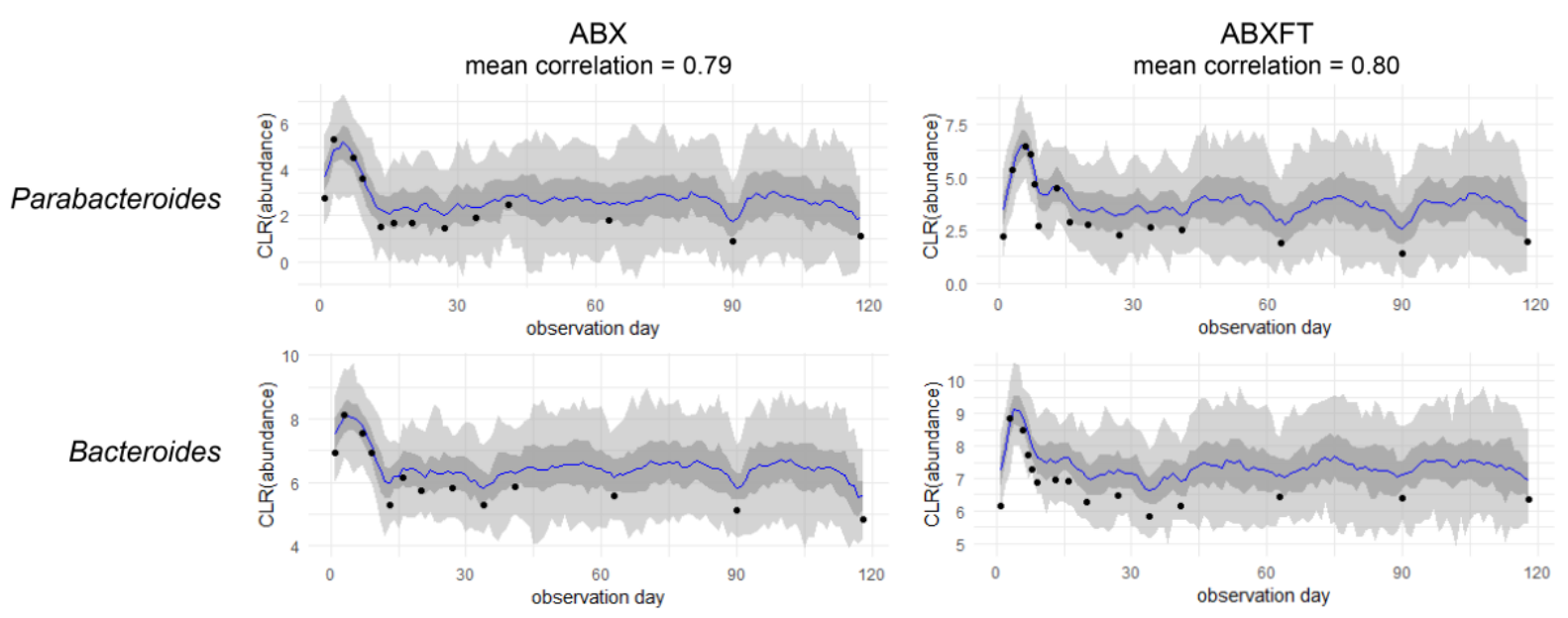

Figure 5. Representative correlation plots for the association between the Centered Log Ratio (CLR) of Bacteroides and Parabacteroides abundances in healthy, male ring-tailed lemurs (Lemur catta) either treated with antibiotics only (ABX) or with antibiotics plus fecal transfaunation (ABXFT). The antibiotic treatment period spans day $0-6$, fecal transfaunation was administered on day 7 , and all days thereafter constitute the period of recovery.

209 via shared ABR genes.

210 During the recovery phase, the gut microbiomes of ABX animals retained only 20 (18

211 positive and 2 negative) of the original 35 strong associations (Table S2, Figure 4c), likely

212 reflecting the paucity of microbes that survived antibiotic treatment. By contrast, the gut

213 microbiomes of ABXFT animals retained the same 31 (23 positive and 8 negative) strong

214 associations (Table S2; Figure 4d), likely reflecting the reintroduction of baseline microbes, and

215 their associations, through fecal transfaunation. Only two associations were shared between

216 ABX and ABXFT animals during recovery: Parabacteroides and Bacteroides, and 
Christensenellaceae R-7 group and Ruminococcaceae NK4A214 group, both of which were also

218 shared during the entire experimental period. Despite variability across treatment groups and

219 phases, some of the strongest associations persisted during recovery (Table S2), further

220 supporting the 'key-stone species' hypothesis.

221

222 Cross-sectional and longitudinal ABR

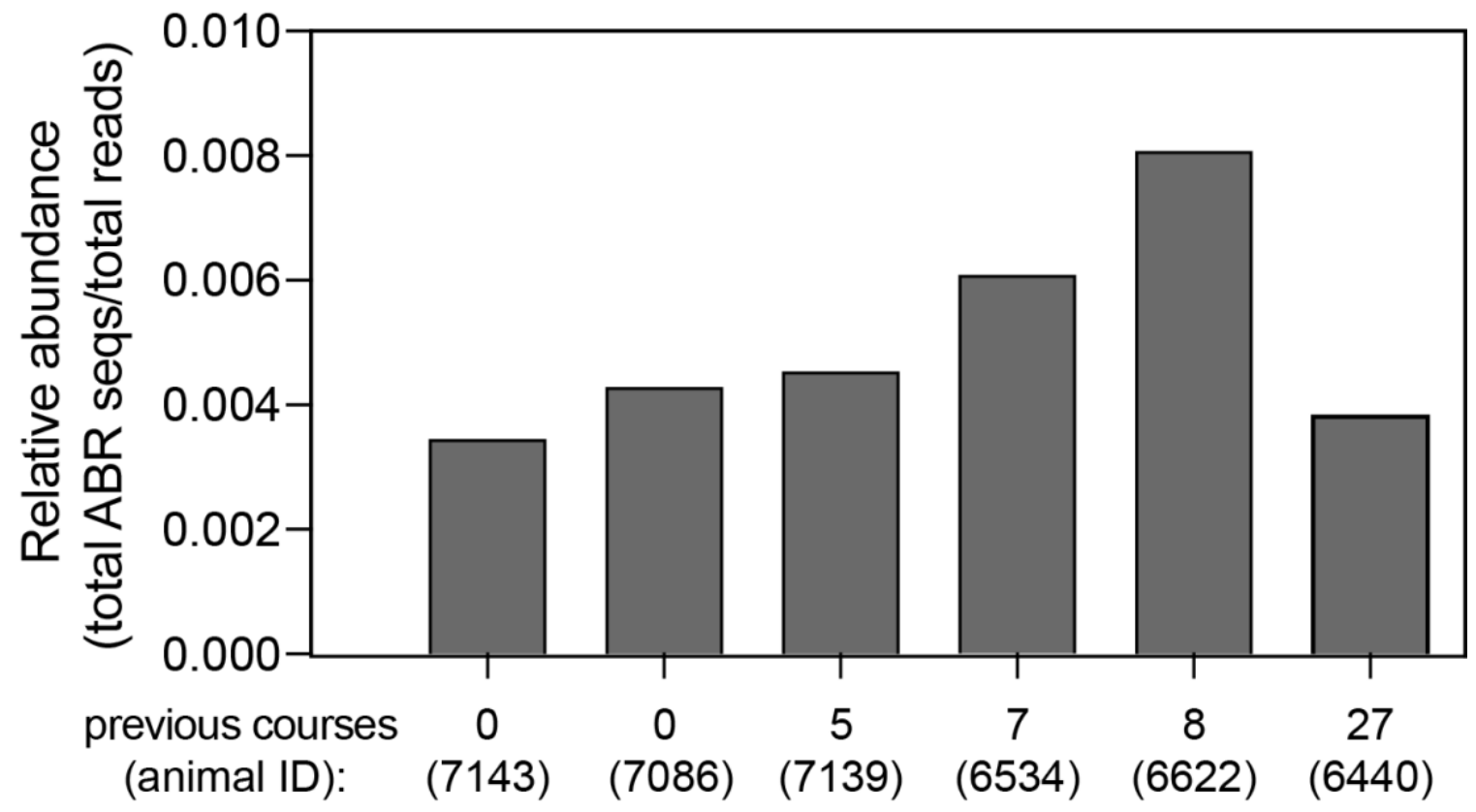

Figure 6. Relative abundance of antibiotic resistance (ABR) genes in six healthy, male ringtailed lemurs (Lemur catta) that had received different numbers of treatment courses of antibiotics across their lifetime.

Across the 30 fecal samples selected for shotgun sequencing (from a subset of subjects),

2243.2 million sequences were assigned to 83 known ABR genes. On average, the majority of the

225 ABR genes detected belonged to four resistance gene families: Tetracycline (51.4\%), Beta-

226 lactam (29.5\%), Aminoglycoside (7.9\%), and Macrolide (1.2\%). There was also minor (>1\%)

227 representation of genes in the Vancomycin, Multi-Drug Resistant, and Sulphonamide families. 
The cross-sectional data on the six, focal animals revealed unexpected variation in ABR.

229 Notably, the two animals (IDs 7143 and 7086) that had never been treated with antibiotics

230 nevertheless harbored ABR at levels similar to those of animals that had previously received

231 numerous courses of antibiotics (Figure 6). Additionally, the animal (ID 6440) with the most

232 numerous antibiotic treatments ( $n=27$ courses), harbored the second lowest abundance of ABR

233 genes, similar to that of the animals with no previous treatment (Figure 6). 


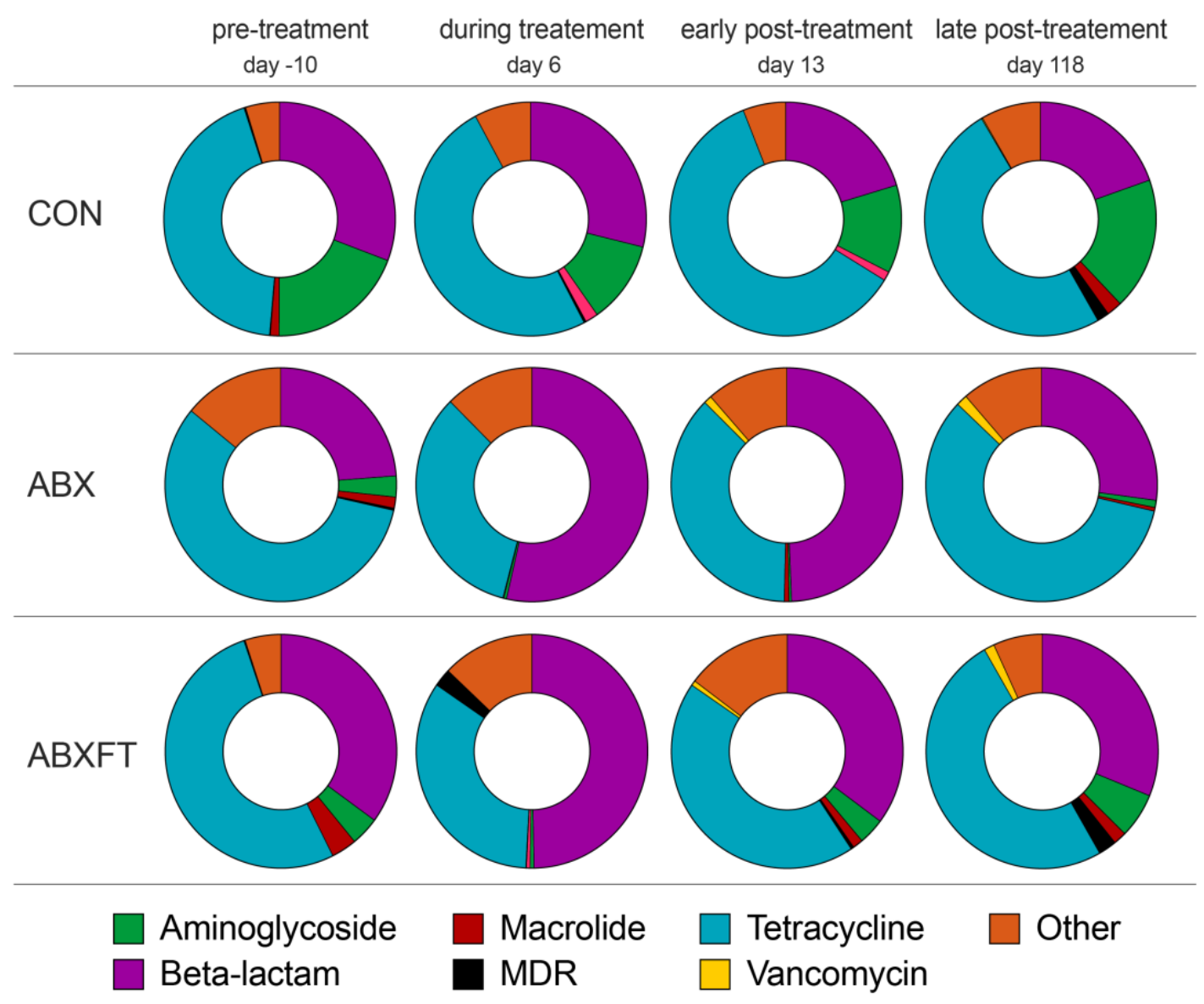

Figure 7. Proportions of antibiotic resistance (ABR) genes identified in healthy, male ring-tailed lemurs (Lemur catta) that received no treatment (CON), antibiotics only (ABX), or antibiotics plus fecal transfaunation (ABXFT). Shown are color-coded resistance gene families at four time points during the study, during which antibiotic treatment was administered on days 0-6 and fecal transfaunation was administered on day 7. MDR = Multi-Drug Resistant.

In analyses of longitudinal variation in the relative abundance of $\mathrm{ABR}$ genes, we found no significant effects of experimental condition or time point (HGAM: experimental condition, F $=0.530, \mathrm{p}=0.603 ;$ time point, $\mathrm{F}=0.602, \mathrm{p}=0.627)$. By contrast, experimental condition significantly affected the proportion of ABR genes assigned to the beta-lactam resistance family.

238 Compared to CON animals, ABX and ABXFT animals harbored significantly greater proportions of beta-lactam resistance genes (HGAM: CON vs. ABX, $t=2.352, p=0.040, C O N$ vs. $\mathrm{ABXFT}, \mathrm{t}=2.439, \mathrm{p}=0.034)$, reflecting the impact of treatment with a beta-lactam antibiotic

241 (amoxicillin). An animal's number of previous antibiotic courses was significantly, but 


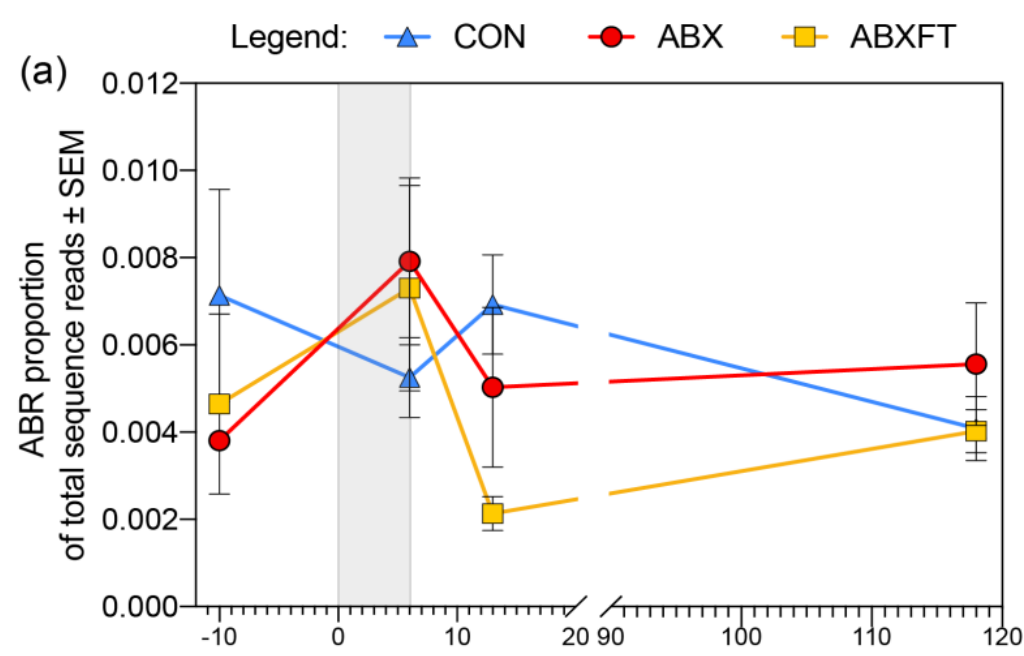

(b)

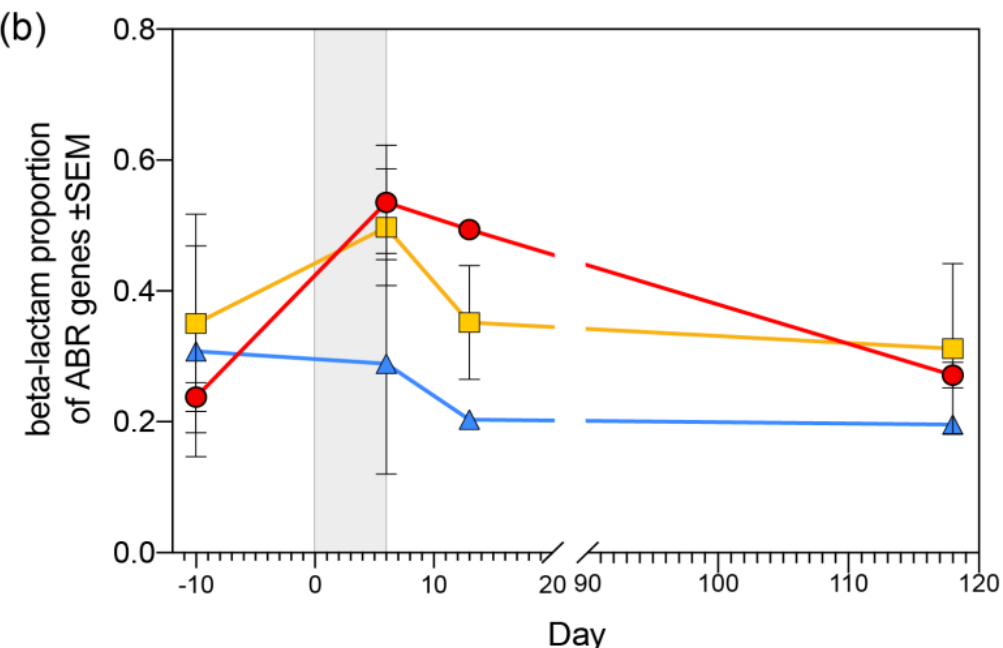

Figure 8. Patterns in antibiotic resistance across healthy, male ring-tailed lemurs (Lemur catta) that received no treatment $(\mathrm{CON})$, antibiotics only (ABX), or antibiotics plus fecal transfaunation (ABXFT). Shown is variation over time in (a) the relative abundance of antibiotic resistance (ABR) genes and (b) the proportion of ABR genes assigned to the betalactam resistance gene family. The gray shaded window represents the period of antibiotic treatment, with the prior period representing baseline and the subsequent period representing recovery. $\mathrm{SEM}=$ standard error of the means.

242 negatively, related to the proportion of beta-lactam genes (HGAM: $t=-2.766, p=0.019$ ). In

$243 \mathrm{ABX}$ and ABXFT animals, samples collected pretreatment and late post-treatment had

244 significantly fewer ABR genes than did samples collected during treatment (HGAM: during vs.

245 pretreatment, $\mathrm{t}=-4.616, \mathrm{p}=0.003$; during vs. late post-treatment, $\mathrm{t}=-4.605, \mathrm{p}=0.006$ ).

246 Although not statistically significant, the proportion of beta-lactam resistance genes during early

247 post-treatment tended to be greater in ABX animals compared to ABXFT animals (HGAM:

248 period and experiment group interaction effect, $\mathrm{t}=1.766, \mathrm{p}=0.127$; Figure 8 ), which may hint at

249 a potential mitigating effect of fecal transfaunation on the persistence of ABR in lemur gut

250 microbiomes. 


\section{Discussion}

253 In our longitudinal experiment, we contribute to the ecological framework for interpreting

254 the dynamics of host-associated microbiomes by tracking bacterial recovery following antibiotic-

255 mediated dysbiosis in healthy nonhuman primates. We provide support for both the 'diversity

256 begets stability' and 'key-stone species' hypotheses, but with different schedules: microbial

257 alpha diversity rebounded quickly (albeit incompletely) in treated animals, whereas beta

258 diversity reflected a trajectory of long-term, microbial instability in animals that received

259 antibiotics alone. The effects of fecal transfaunation on recovery of alpha diversity were

260 negligeable, but for beta diversity, this procedure hastened and stabilized the recovery of

261 community composition, supporting fecal transfaunation as an effective tool for treating

262 microbial dysbiosis. The associations between bacteria varied between experimental conditions,

263 suggesting that the relationships between microbial groups may have contributed to the

264 differential effects of $\mathrm{ABX}$ and $\mathrm{ABXFT}$ treatments, which could have been governed by the

265 presence of ABR genes. Our cross-sectional analysis of ABR showed that the prevalence of

266 ABR genes in a host is not necessarily correlated with that host's previous exposure to

267 antibiotics; ABR can be acquired and maintained in the gut microbiomes of lemurs that had no

268 previous antibiotic treatment. Longitudinally, ABR gene profiles reflected the type of antibiotic

269 being used. As expected, the proportion of ABR genes that confer resistance to beta-lactamase

270 antibiotics increased during treatment with amoxicillin. Lastly, fecal transfaunation may mitigate

271 the persistence of ABR during recovery from antibiotic treatment. Using a holistic and

272 longitudinal approach, across scales, allowed elucidating microbial dynamics that otherwise

273 would have been imperceptible. 
Consistent with previous findings ${ }^{56,57}$, and with the known efficacy of amoxicillin as a broad-

275 spectrum antibiotic ${ }^{58}$, we found that animals receiving antibiotics concurrently showed a drastic

276 decrease in alpha diversity. Contrary to expectations, however, lemurs post treatment showed a

277 rapid rebound in alpha diversity, with $\mathrm{ABX}$ and ABFT animals showing no significant difference

278 in their recovery trajectories. The rapidity of these patterns may owe to the healthy status of the

279 hosts and to the relatively short period of antibiotic treatment. The ability to recolonize or re-

280 diversify a microbiome after dysbiosis can be severely dampened by injury or disease ${ }^{59,60}$ and by

281 recurrent antibiotic treatment ${ }^{61,62}$. Or, as omnivores with a broad dietary range, ring-tailed

282 lemurs may have shown more rapid recovery than would be observed by dietary specialists ${ }^{42}$.

283 Beyond external influences (e.g. from diet, the physical environment, or social interaction), alpha

284 diversity can also increase from within. Indeed, even if antibiotic treatment causes local bacterial

285 extirpations, certain taxa can persist either by expressing ABR genes ${ }^{8,63}$ or by sequestering in

286 areas of the gastrointestinal tract that are less affected by antibiotics (i.e., the appendix or

287 cecum $^{64,65}$ ), allowing for in-kind recolonization after disruption. Nevertheless, as evidenced by

288 the results on beta diversity, early recovery of alpha diversity did not entail strictly in-kind

289 recolonization of lemur gut microbiota. The rapidity in these patterns could thus lend support to

290 the 'diversity begets stability' hypothesis, in that the key first step in community restoration may

291 be to regain diversity, regardless of microbial composition.

292 Patterns of beta diversity elucidated longer-term effects of antibiotics on gut microbiome

293 community composition. Throughout the four-month recovery period of ABX animals, the

294 microbial community did not return to the baseline composition or even reach an alternative

295 stable state. In line with previous evidence and with the key-stone species hypothesis, this pattern

296 indicated that recouping key microbial members may be more elusive (and perhaps more critical 
297 to stability) than recouping sheer numbers of taxa ${ }^{55}$. Indeed, as predicted under this hypothesis,

298 fecal transfaunation showed a stabilizing effect on community composition. The bacterial

299 associations that characterized the recovery phase in ABXFT animals were more numerous and

300 almost wholly different from those in ABX lemurs, suggesting that multitudinous microbial

301 interactions underpin some of the effects of fecal transfaunation. These findings are consistent

302 with the concept of competitive exclusion, whereby the diverse group of reintroduced, native

303 bacteria outcompete pathogenic or opportunistic microbes ${ }^{66,67}$. Although there is much to learn

304 about the modes of action in successful transfaunation, we add to the mounting evidence that

305 they are a promising tool to hasten recovery from dysbiosis ${ }^{68-70}$.

306 Of the bacterial associations present in the two treatment conditions, Parabacteroides and

307 Bacteroides - two, closely related taxa with similar functional potential ${ }^{71}$ - dominated the

308 observed relationships. The strength and quantity of relationships between Bacteroides and other

309 bacterial taxa support the foundational membership of Bacteroides in the lemur gut

310 microbiome ${ }^{42,72,73}$. Notably, increases in the log ratio abundance of Bacteroides during antibiotic

311 treatment indicated that its members maintained or increased their relative abundances while

312 other taxa were eliminated. Indeed, the Bacteroides genus is notorious for showing ABR. The

313 diversity of its resistance mechanisms ${ }^{74,75}$, coupled with extensive lateral gene transfer within

314 members of the genus and with non-Bacteroides taxa ${ }^{76,77}$, contributed to certain Bacteroides spp.

315 having one of the highest resistance rates among known anaerobic pathogens ${ }^{78}$. Furthermore,

316 certain Bacteroides harbor an unknown molecular mechanism that confers resistance specifically

317 to amoxicillin ${ }^{79}$. Certain Bacteroides strains can even shield other taxa from the effects of beta-

318 lactam antibiotics ${ }^{80}$. Combined with this evidence, our results suggest that Bacteroides in the 
lemur gut microbiome likely have amoxicillin resistance mechanisms that enable them to dictate most bacterial relationships during recovery from antibiotic-induced dysbiosis.

ABR genes, including some that are considered clinically relevant ${ }^{81}$, were present within the

322 gut microbiome of all lemurs. Somewhat surprisingly, lemurs that had never received antibiotic

323 treatment showed resistance levels similar to those of lemurs previously treated with antibiotics.

324 Researchers have shown that bacteria and their genes can be shared between hosts that

325 cohabitate $^{82,83}$ or share social partners ${ }^{84}$. Furthermore, ABR genes often reside on mobile genetic

326 units and are prone to rapid transfer between microbes ${ }^{85}$. Indeed, the 'resistance crisis' is

327 perpetuated by the ubiquitous spread of ABR genes around the world ${ }^{10,86,87}$. Here, we find that

328 lemurs are not exempt from these phenomena and, for captive animals especially, ABR could

329 pose a severe threat to animal health ${ }^{88,89}$. Methods to mitigate the development and spread of

$330 \mathrm{ABR}$ among animal populations, including perhaps via fecal transfaunation, may prove to be a

331 critical facet of combatting the resistance crisis ${ }^{86,90}$.

332 Collectively, these results further our understanding of host-microbe relationships in the

333 Anthropocene era ${ }^{91,92}$. Evolutionary medicine is founded on the principle that, over evolutionary

334 and proximate scales, changing environments and conditions influence aspects of well-being ${ }^{93-95}$.

335 As scientists and medical practitioners increasingly recognize the value of a One Health

336 perspective on human medicine (i.e., that human, animal, and environmental health are

337 inseparable), holistic approaches will add context to our understanding of clinically relevant

338 phenomena (i.e., the effects of antibiotics and ABR; ${ }^{87,96,97}$ ). Ultimately, shedding light on how

339 'old friends' react to aspects of the 'new world' is relevant both to our understanding of the

340 evolution of symbiosis and to its implications for modern medicine. 


\section{Methods}

\section{Study subjects and housing}

345 Our study subjects were 11 healthy, reproductively intact, adult (4-16 yrs old), male ring-

346 tailed lemurs housed in 10 conspecific, mixed-sex groups at the Duke Lemur Center (DLC;

347 Durham, NC, USA). Within a two-year period, 10 of them underwent a control round with no 348 treatment, but all 11 underwent one round of antibiotic treatment (see below), while living in 349 their same social groups. During inclement weather $\left(<5^{\circ} \mathrm{C}\right)$, the groups would be sequestered in 350 indoor enclosures, otherwise, they all had access to indoor and outdoor enclosures

351 (approximately $146 \mathrm{~m}^{2} / \mathrm{animal}$ ). Some of the groups additionally had access to large, forest 352 enclosures where they semi-free-ranged with heterospecific lemurs. The animals received a diet 353 of produce and commercial primate chow and, while semi-free-ranging, had access to natural 354 foods foraged from the forest. Additional information on the lemurs' diet, foraging, and social 355 behavior have been reported elsewhere ${ }^{98}$. The subjects were maintained in accordance with the 356 NIH Guide for the Care and Use of Laboratory Animals, and procedures were approved by the 357 Institutional Animal Care and Use Committee of Duke University (protocol A111-16-05).

Study design and sample collection

To allow for a partial cross-over design, we conducted the study during two matched

361 periods (October-February) in consecutive years, during the subjects' breeding season in the

362 Northern Hemisphere ${ }^{99}:$ 2016-2017 (Y1, $\mathrm{n}=10$ subjects) and 2017-2018 (Y2, $\mathrm{n}=11$ subjects).

363 In each year, we assigned the subjects to one of three experimental groups: control animals

$364(\mathrm{CON} ; \mathrm{Y} 1, \mathrm{n}=4 ; \mathrm{Y} 2, \mathrm{n}=6)$, antibiotic-treated animals $(\mathrm{ABX} ; \mathrm{Y} 1, \mathrm{n}=3 ; \mathrm{Y} 2, \mathrm{n}=3)$, and 
antibiotic-treated animals receiving a fecal transfaunation (ABXFT; Y1, $\mathrm{n}=3 ; \mathrm{Y} 2, \mathrm{n}=2$ ). To avoid administering antibiotics twice to any animal, each animal was assigned to the CON group

367 in one of the two years.

368 Each year of study involved three phases, lasting a total of $\sim 125$ days: a pretreatment or 369 baseline phase (lasting $\sim 6$ days; i.e., day -6 to -1 ), a treatment phase (lasting 7-8 days; i.e., day 0 370 to $6 / 7$ ), and a recovery phase (lasting $~ 110$ days). In the treatment phase, all treated animals

$371(\mathrm{ABX}$ and ABXFT; $\mathrm{n}=11)$ received a 7-day course of the broad-spectrum, beta-lactam

372 antibiotic, amoxicillin (10 mg/kg body weight, received orally, twice daily). Approximately 24

373 hrs after completion of the full antibiotic regimen, ABXFT subjects received a fecal

374 transfaunation consisting of their own feces: 2-3 fecal pellets, collected pretreatment, were mixed

375 with water and administered orally via syringe or feeding tube, according to routine procedures

376 that have been adopted by the DLC since the mid 1980s to treat outbreaks of gastrointestinal

377 diseases $45,100,101$.

378 The phases were additionally differentiated by the frequency with which we collected fecal

379 samples: We collected samples every 1-3 days before, during, and immediately after the

380 treatment phase, after which sampling occurred every 5-28 days. Typically, upon the subject's

381 morning voiding, between 7:00 am and 11:30 am, we opportunistically collected fresh fecal

382 samples. On occasion, we collected samples from awake, gently restrained animals that were

383 habituated to capture and collection procedures. At each time point, we sampled all subjects and

384 we maintained analogous sampling regimes across years. We collected all samples in sterile, 15-

$385 \mathrm{ml}$ falcon tubes, immediately placed them on ice, and then stored them in a $-80{ }^{\circ} \mathrm{C}$ freezer within 3862 hours, until analysis. 
Microbial DNA extraction, sequencing and bioinformatics

389

Using the DNeasy Powersoil kit (QIAGAN, Frederick, MD), we extracted microbial

gDNA from fecal samples and from four blank controls, to control for possible contamination.

391 We quantified the extracted gDNA using a Fluorometer (broad-spectrum kit, Qubit 4, Thermo

392 Fisher Scientific, Waltham, MA). These extractions were used for bacterial identification (via

393 16S rRNA amplicon sequencing) and ABR gene identification (via shotgun sequencing), as

394 described below.

395

396

Bacterial identification

397 We shipped aliquots of extracted gDNA to the Argonne National Laboratory's

398 Environmental Sequencing facility (Lemont, IL) for library preparation and sequencing of the

399 16S rRNA gene. There, the V4 region of the 16S rRNA gene (515F-806R) was amplified with

400 region-specific primers adapted for the Illumina MiSeq platform ${ }^{102}$. Forward primers contained

401 a 12-base barcode sequence to support pooling of samples in each flow cell lane. Once pooled,

402 amplicon libraries were cleaned using AMPure XP Beads (Beckman Coulter, Pasadena, CA),

403 and quantified using a fluorometer (Qubit 4). Amplicons were sequenced on a 2 x $151 \mathrm{bp}$

404 Illumina MiSeq run ${ }^{102}$. Sequencing reads are available on the National Center for Biotechnology

405 Information's Sequence Read Archive (BioProject ID \#TBD, BioSample accession \#s TBD).

406 In collaboration with Duke University’s Genomic Analysis and Bioinformatics Shared

407 Resource, 16S raw sequence data were analyzed using a bioinformatics pipeline generated in

408 QIIME2 (ver 2018.11) ${ }^{103}$. The pipeline included steps to join, demultiplex, and quality-filter

409 sequence reads. The DADA2 plugin (q2-dada2) ${ }^{104}$ was used to denoise, quality-filter, and

410 remove phiX and chimeric sequences from the demultiplexed reads. Using the resulting 
411 sequences, we discarded samples with $<10,000$ reads. To determine taxonomic classification, we

412 used a pre-trained Naive Bayes classifier at 99\% sequence identity (SILVA-132 database) ${ }^{105,106 .}$

413 After bioinformatic processing, a total of 344 fecal samples (from all subjects across all study

414 phases) yielded over 23.4 million 16S sequences (mean per sample $=59,766)$.

415 We used the feature tables and taxonomies of bacterial members to calculate Shannon-

416 Weaver diversity (i.e., alpha diversity). To assess microbial composition (i.e., beta diversity), we

417 calculated unweighted UniFrac, a distance metric that considers the phylogenetic relationships

418 between taxa. After calculating these diversity metrics, we combined features without assigned

419 taxonomy below the Kingdom level into an "Unassigned" category. We also included the

420 conglomerate "Other" to represent the rare taxa that had relative abundances $<1 \%$.

421

422 ABR identification

423 To allow for cross-sectional and longitudinal analyses of ABR genes, while limiting the

424 expense of metagenomic analyses, we performed shotgun sequencing on samples from six

425 lemurs, two per experimental group, that ranged in their previous exposure to antibiotics (0-27

426 previous courses). For cross-sectional analysis, we included one sample from each subject's

427 pretreatment phase in Y1. For longitudinal analysis (which we prioritized), we included four

428 samples from each animal (days -10, 6, 13, and 118) in Y2. We shipped this subset of extractions

429 ( $\mathrm{n}=30)$ for shotgun sequencing to CosmosID (Rockville, MD), where DNA libraries were

430 prepared using the Illumina Nextera XT library preparation kit, with a modified protocol. Library

431 quantity was assessed with Qubit (ThermoFisher). Libraries were then sequenced on an Illumina

432 HiSeq platform 2 x $150 \mathrm{bp}$. 
433 The samples selected for shotgun sequencing averaged 20.4 million sequences per sample.

434 The resulting unassembled sequencing reads underwent multi-kingdom microbiome analysis and

435 profiling of antibiotic resistance genes using the CosmosID bioinformatics platform (CosmosID

436 Inc., Rockville, MD), as described elsewhere ${ }^{107,108}$. The antibiotic resistance and virulence genes

437 in the microbiome were identified by querying the unassembled sequence reads against the

438 CosmosID-curated antibiotic resistance and virulence associated gene databases ${ }^{109,110 .}$

\section{Statistical analyses}

441 To characterize variation in bacterial diversity and composition, we used Hierarchical

442 General Additive Models (HGAM) ${ }^{111}$, which have the flexibility to accommodate nonlinear

443 trends (See Supplementary Materials 1 for full model syntax). We used this model to test for

444 patterns in bacterial alpha and beta diversity. For analyses of beta diversity, we first used

445 Principal Coordinates Analysis (PCoA) to visualize variation in bacterial composition (UniFrac

446 distance) in coordinate space. Subsequently, we used distance metrics to calculate change in

447 bacterial composition relative to a pretreatment, baseline community (collected 4 days before the 448 onset of treatment for all animals; QIIME2). We tested for variation in these calculated distance

449 measures using our HGAM. To assess the response to and recovery from treatment, we first used

450 our models to test for variation across the entire dataset and, then, using subsets of the alpha and

451 beta diversity data, we focused our model on the treatment and recovery phases.

452 To better understand the short- and longer-term process of recovery, we tested for

453 associations between bacterial taxa over time and evaluated how these associations may have

454 differed between treatment groups. To exclude spurious associations, we first removed a few

455 pretreatment samples from each animal's series, further allowing us to focus on associations 
456 during the treatment and recovery phases. To reduce sparsity in the dataset and ease the

457 computational burden, we removed rare taxa present in < five samples across the full dataset,

458 clustered taxa at the genus level, and grouped as 'Other' all low-abundance genera with $<0.01 \%$

459 of total counts. This filtering removed $<1 \%$ of total sequence counts.

460 To naturally model the irregular temporal spacing in the observations and manage

461 autocorrelation between samples, we fitted a Bayesian multivariate Gaussian process to each of

462 multiple synthetic replicates of the dataset (see resampling procedure below). We then inferred a

463 distribution over the covariance between microbes. The sample collection schedule motivated

464 two key choices in noise modeling and data representation. First, because stochasticity exists in

465 sample collection, processing, and sequencing, we used a resampling method similar to that of

466 ALDEx2 $2^{112,113}$ to emulate the variation that would be expected from replicate measurements.

467 Second, to account for the compositional nature of the sequence count data within our model ${ }^{114}$,

468 we used log ratios as compositional representations of the data. We converted the estimated

469 covariance matrices to correlation matrices and thresholded all pairwise correlations between

470 microbes to select as significant those with $95 \%$ credible intervals that excluded zero correlation

471 (i.e., those with strong positive or negative associations). We then ranked associations by their

472 median strength and selected those in excess of correlation $\rho>0.5$ or $\rho<-0.5$ as strong

473 associations.

474 Because our cross-sectional ABR data had small sample sizes and minimal statistical power,

475 we were limited to examining qualitative trends. To assess patterns in longitudinal ABR data, we

476 used another HGAM model (Supplementary Materials 1), fitted to the following aspects of ABR

477 gene profiles: Across all three experimental groups, we tested for patterns in the relative

478 abundance of all sequences that were assigned to an ABR gene and in the proportion of ABR 
479 genes assigned to beta-lactam resistance genes. To further investigate the effects of antibiotics

480 and fecal transfaunation on ABR, we ran additional HGAMs on the ABR data from only the

481 ABX and ABXFT groups.

482

483 Acknowledgements

484 We thank the current and past staff members of the DLC, particularly David Brewer, Erin 485 Ehmke, Megan Davison, Cat Ostrowski, Bobby Schopler, Melanie Simmons, and Kay Welser,

486 for their insights and help with animal handling. Conversations with Lydia Greene were

487 instrumental during this project's inception. We are grateful to Sarah Owens at Argonne National

488 Laboratory and Karlis Graubics and Brian Fanelli at CosmosID provided guidance and

489 sequencing services. David Corcoran and Zhengzheng Wei at the Duke Genomic Analysis and

490 Bioinformatics Shared Resource provided bioinformatics analyses. Funding was provided by

491 awards from the National Science Foundation (BCS 1749465 to CD), DLC Director's Fund (to

492 SB and RH), and Duke Microbiome Center (formerly Duke Center for the Genomics of

493 Microbial Systems, Grants-In-Aid for Microbiome Bioinformatic Analysis to CD). This is DLC

494 publication number (\#\#TBD).

495

\section{Statement of competing interests}

497 We attest that no author has financial or non-financial competing interests.

References

5001 Koskella B, Bergelson J. The study of host-microbiome (co) evolution across levels of $501 \quad$ selection. Philos Trans R Soc B 2020; 375: 20190604. 
5022 Lynch JB, Hsiao EY. Microbiomes as sources of emergent host phenotypes. Science (80- )

$503 \quad 2019 ; 365:$ 1405-1409.

5043 Brown K, DeCoffe D, Molcan E, Gibson DL. Diet-induced dysbiosis of the intestinal

505 microbiota and the effects on immunity and disease. Nutrients 2012; 4: 1095-1119.

5064 Li J, Zhao F, Wang Y, Chen J, Tao J, Tian G, Wu S, Liu W, Cui Q, Geng B. Gut microbiota

507 dysbiosis contributes to the development of hypertension. Microbiome 2017; 5: 1-19.

5085 Moloney RD, Desbonnet L, Clarke G, Dinan TG, Cryan JF. The microbiome: stress, health $509 \quad$ and disease. Mamm Genome 2014; 25: 49-74.

5106 Rook GAW. 99th Dahlem conference on infection, inflammation and chronic inflammatory

511 disorders: Darwinian medicine and the 'hygiene'or 'old friends' hypothesis. Clin Exp

$512 \quad$ Immunol 2010; 160: 70-79.

5137 Rook GAW, Martinelli R, Brunet LR. Innate immune responses to mycobacteria and the 514 downregulation of atopic responses. Curr Opin Allergy Clin Immunol 2003; 3: 337-342.

5158 Francino MP. Antibiotics and the human gut microbiome: dysbioses and accumulation of $516 \quad$ resistances. Front Microbiol 2016; 6: 1-11.

5179 Langdon A, Crook N, Dantas G. The effects of antibiotics on the microbiome throughout 518 development and alternative approaches for therapeutic modulation. Genome Med 2016; 8:

$519 \quad 39$.

52010 Ventola CL. The antibiotic resistance crisis: part 1: causes and threats. Pharm Ther 2015;

$521 \quad$ 40: 277.

52211 Taur Y, Coyte K, Schluter J, Robilotti E, Figueroa C, Gjonbalaj M, Littmann ER, Ling L,

523 Miller L, Gyaltshen Y et al. Reconstitution of the gut microbiota of antibiotic-treated

524 patients by autologous fecal microbiota transplant. Sci Transl Med 2018; 10. 
52512 D’Costa VM, King CE, Kalan L, Morar M, Sung WWL, Schwarz C, Froese D, Zazula G,

526 Calmels F, Debruyne R et al. Antibiotic resistance is ancient. Nature 2011; 477: 457-461.

52713 Davies JE. Origins, acquisition and dissemination of antibiotic resistance determinants. In:

528 Chadwick DJ, Goode JA (eds). Antibiotic Resistance: Origins, Evolution, Selection and

$529 \quad$ Spread. Wiley Online Library, 1997, pp 15-27.

53014 Atwood KC, Schneider LK, Rryan FJ. Selective mechanisms in bacteria. In: Cold Spring

531 Harbor Symposia on Quantitative Biology. Cold Spring Harbor Laboratory Press, 1951, pp

$532 \quad 345-355$.

53315 Denamur E, Matic I. Evolution of mutation rates in bacteria. Mol Microbiol 2006; 60: 820-

534827.

53516 Ochman H, Lawrence JG, Groisman E. Lateral gene transfer and the nature of bacterial 536 innovation. Nature 2000; 405: 299-304.

53717 Thomas CM, Nielsen KM. Mechanisms of, and barriers to, horizontal gene transfer between $538 \quad$ bacteria. Nat Rev Microbiol 2005; 3: 711-721.

53918 Allen HK, Donato J, Wang HH, Cloud-Hansen KA, Davies J, Handelsman J. Call of the 540 wild: antibiotic resistance genes in natural environments. Nat Rev Microbiol 2010; 8: 251541259.

54219 Aminov RI. The role of antibiotics and antibiotic resistance in nature. Environ Microbiol $543 \quad 2009 ; 11: 2970-2988$.

54420 Ferrer M, Méndez-García C, Rojo D, Barbas C, Moya A. Antibiotic use and microbiome 545 function. Biochem Pharmacol 2017; 134: 114-126.

54621 Cho I, Yamanishi S, Cox L, Methe BA, Zavadil J, Li K, Gao Z, Mahana D, Raju K, Teitler I 547 et al. Antibiotics in early life alter the murine colonic microbiome and adiposity. Nature 
2012; 488: 621-626.

22 Zarrinpar A, Chaix A, Xu ZZ, Chang MW, Marotz CA, Saghatelian A, Knight R, Panda S et al. Antibiotic-induced microbiome depletion alters metabolic homeostasis by affecting gut signaling and colonic metabolism. Nat Commun 2018; 9: 1-13.

55223 Kolář M, Urbánek K, Látal T. Antibiotic selective pressure and development of bacterial $553 \quad$ resistance. Int J Antimicrob Agents 2001; 17: 357-363.

55424 Witte W. Selective pressure by antibiotic use in livestock. Int J Antimicrob Agents 2000; 16:

$555 \quad 19-24$.

55625 Stokes HW, Gillings MR. Gene flow, mobile genetic elements and the recruitment of

557 antibiotic resistance genes into Gram-negative pathogens. FEMS Microbiol Rev 2011; 35 :

$558 \quad 790-819$.

55926 Weber DJ, Raasch R, Rutala WA. Nosocomial infections in the ICU: the growing 560 importance of antibiotic-resistant pathogens. Chest 1999; 115: 34S-41S.

56127 Britton RA, Young VB. Interaction between the intestinal microbiota and host in 562 Clostridium difficile colonization resistance. Trends Microbiol 2012; 20: 313-319.

56328 Stecher B, Maier L, Hardt W-D. 'Blooming'in the gut: how dysbiosis might contribute to $564 \quad$ pathogen evolution. Nat Rev Microbiol 2013; 11: 277.

56529 Chambers HF, DeLeo FR. Waves of resistance: Staphylococcus aureus in the antibiotic era. $566 \quad$ Nat Rev Microbiol 2009; 7: 629-641.

56730 Petty NK, Zakour NL Ben, Stanton-Cook M, Skippington E, Totsika M, Forde BM, Phan 568 M-D, Moriel DG, Peters KM, Davies M et al. Global dissemination of a multidrug resistant $569 \quad$ Escherichia coli clone. Proc Natl Acad Sci 2014; 111: 5694-5699.

57031 Aroniadis OC, Brandt LJ. Fecal microbiota transplantation: past, present and future. Curr 
Opin Gastroenterol 2013; 29: 79-84.

572

573

574

575

576

577

578

579

580

581

582

583

584

585

586

587

588

589

590

591

592

593

32 Grehan MJ, Borody TJ, Leis SM, Campbell J, Mitchell H, Wettstein A. Durable alteration of the colonic microbiota by the administration of donor fecal flora. J Clin Gastroenterol 2010; 44: 551-561.

33 Bo T-B, Zhang X-Y, Kohl KD, Wen J, Tian S-J, Wang D-H. Coprophagy prevention alters microbiome, metabolism, neurochemistry, and cognitive behavior in a small mammal. ISME $J$ 2020; 14: 2625-2645.

34 Osawa R, Blanshard WH, Ocallaghan PG. Microbiological studies of the intestinal microflora of the koala, Phascolarctos cinereus. 2. Pap, a special maternal feces consumed by juvenile koalas. Aust J Zool 1993; 41: 611-620.

35 Chaitman J, Jergens AE, Gaschen F, Garcia-Mazcorro JF, Marks SL, Marroquin-Cardona AG, Richter K, Rossi G, Suchodolski JS, Weese JS et al. Commentary on key aspects of fecal microbiota transplantation in small animal practice. Vet Med Res Reports 2016; 7: 71.

36 Niederwerder MC. Fecal microbiota transplantation as a tool to treat and reduce susceptibility to disease in animals. Vet Immunol Immunopathol 2018; 206: 65-72.

37 Bakken JS, Borody T, Brandt LJ, Brill J V, Demarco DC, Franzos MA, Kelly C, Khoruts A, Louie T, Martinelli LP et al. Treating Clostridium difficile infection with fecal microbiota transplantation. Clin Gastroenterol Hepatol 2011; 9: 1044-1049.

38 Brandt LJ, Aroniadis OC, Mellow M, Kanatzar A, Kelly C, Park T, Stollman N, Rohlke F, Surawicz C et al. Long-Term Follow-Up of Colonoscopic Fecal Microbiota Transplant for Recurrent Clostridium difficile Infection. Am J Gastroenterol 2012; 107: 1079-1087.

39 Amato KR, Sanders JG, Song SJ, Nute M, Metcalf JL, Thompson LR, Morton JT, Amir A, McKenzie VJ, Humphrey G et al. Evolutionary trends in host physiology outweigh dietary 
niche in structuring primate gut microbiomes. ISME J 2019; : 1.

59540 Clayton JB, Gomez A, Amato K, Knights D, Travis DA, Blekhman R, Knight R, Leigh S,

596 Stumpf R, Wolf T et al. The gut microbiome of nonhuman primates: Lessons in ecology and 597 evolution. Am J Primatol 2018; : e22867.

59841 Greene LK, Clayton JB, Rothman RS, Semel BP, Semel MA, Gillespie TR, Wright PC, 599 Drea CM. Local habitat, not phylogenetic relatedness, predicts gut microbiota better within 600 folivorous than frugivorous lemur lineages. Biol Lett 2019; 15: 20190028.

60142 Greene LK, Bornbusch SL, McKenney EA, Harris RL, Gorvetzian SR, Yoder AD, Drea 602 CM. The importance of scale in comparative microbiome research: New insights from the 603 gut and glands of captive and wild lemurs. Am J Primatol 2019.

60443 Gould L. Lemur catta Ecology : What We Know and What We Need to Know. In: Gould L, 605 Sauther ML (eds). Lemurs: Ecology and Adaptation. Springer: New York, 2006, pp 255606274.

60744 Jolly A, Sussman RW, Koyama N, Rasamimanana H. Ringtailed lemur biology: Lemur 608 catta in Madagascar. Springer Science \& Business Media, 2006.

60945 McKenney EA, Greene LK, Drea CM, Yoder AD. Down for the count: Cryptosporidium $610 \quad$ infection depletes the gut microbiome in Coquerel's sifakas. Microb Ecol Health Dis 2017; 611 28: 1335165.

61246 Konopka A. What is microbial community ecology? ISME J 2009; 3: 1223-1230.

61347 Moya A, Ferrer M. Functional redundancy-induced stability of gut microbiota subjected to 614 disturbance. Trends Microbiol 2016; 24: 402-413.

61548 Wohl DL, Arora S, Gladstone JR. Functional redundancy supports biodiversity and 616 ecosystem function in a closed and constant environment. Ecology 2004; 85: 1534-1540. 
61749 Johnson KH, Vogt KA, Clark HJ, Schmitz OJ, Vogt DJ. Biodiversity and the productivity

$618 \quad$ and stability of ecosystems. Trends Ecol Evol 1996; 11: 372-377.

61950 McNaughton SJ. Diversity and stability of ecological communities: a comment on the role

620 of empiricism in ecology. Am Nat 1977; 111: 515-525.

62151 Mccann KS. The diversity-stability debate. 2000; 405.

62252 Banerjee S, Schlaeppi K, van der Heijden MGA. Keystone taxa as drivers of microbiome $623 \quad$ structure and functioning. Nat Rev Microbiol 2018; 16: 567-576.

62453 Berry D, Widder S. Deciphering microbial interactions and detecting keystone species with 625 co-occurrence networks. Front Microbiol 2014; 5: 219.

62654 Fisher CK, Mehta P. Identifying keystone species in the human gut microbiome from 627 metagenomic timeseries using sparse linear regression. PLoS One 2014; 9: e102451.

62855 Gibbons SM. Keystone taxa indispensable for microbiome recovery. Nat Microbiol 2020; 5: $629 \quad 1067-1068$.

63056 Palleja A, Mikkelsen KH, Forslund SK, Kashani A, Allin KH, Nielsen T, Hansen TH, Liang 631 S, Feng Q, Zhang C et al. Recovery of gut microbiota of healthy adults following antibiotic $632 \quad$ exposure. Nat Microbiol 2018; 3: 1255-1265.

63357 Vlčková K, Gomez A, Petrželková KJ, Whittier CA, Todd AF, Yeoman CJ, Nelson KE, 634 Wilson BA, Stumpf RM, Modrý D et al. Effect of antibiotic treatment on the 635 gastrointestinal microbiome of free-ranging western lowland gorillas (Gorilla g. gorilla). $636 \quad$ Microb Ecol 2016; 72: 943-954.

63758 Kaur SP, Rao R, Nanda S. Amoxicillin: a broad spectrum antibiotic. Int J Pharm Pharm Sci $638 \quad 2011 ; 3: 30-37$.

63959 Krezalek MA, Alverdy JC. The role of the microbiota in surgical recovery. Curr Opin Clin 
Nutr Metab Care 2016; 19: 347-352.

64160 Nicholson SE, Merrill D, Zhu C, Burmeister DM, Zou Y, Lai Z, Darlington DN, Lewis AM,

642 Newton L, Scroggins S et al. Polytrauma independent of therapeutic intervention alters the

643 gastrointestinal microbiome. Am J Surg 2018; 216: 699-705.

64461 McDonald LC. Effects of short-and long-course antibiotics on the lower intestinal

645 microbiome as they relate to traveller's diarrhea. J Travel Med 2017; 24: S35-S38.

64662 Korpela K, Salonen A, Virta LJ, Kekkonen RA, Forslund K, Bork P, De Vos WM. Intestinal

647 microbiome is related to lifetime antibiotic use in Finnish pre-school children. Nat Commun

$648 \quad 2016 ; 7: 10410$.

64963 Van Schaik W. The human gut resistome. Philos Trans R Soc B Biol Sci 2015; 370:

65020140087.

65164 Bollinger RR, Barbas AS, Bush EL, Lin SS, Parker W. Biofilms in the normal human large

652 bowel: fact rather than fiction. Gut 2007; 56: 1481-1482.

65365 Laurin M, Everett M Lou, Parker W. The cecal appendix: one more immune component 654 with a function disturbed by post-industrial culture. Anat Rec Adv Integr Anat Evol Biol $655 \quad 2011 ; 294: 567-579$.

65666 Willing BP, Jansson JK. The gut microbiota: ecology and function. Lawrence Berkeley 657 National Lab, Berkeley, CA (United States), 2010.

65867 Zeng W, Shen J, Bo T, Peng L, Xu H, Nasser MI, Zhuang Q, Zhao M. Cutting edge:

659 Probiotics and fecal microbiota transplantation in immunomodulation. J Immunol Res 2019;

6602019.

66168 Jin Song S, Woodhams DC, Martino C, Allaband C, Mu A, Javorschi-Miller-Montgomery

662 S, Suchodolski JS, Knight R. Engineering the microbiome for animal health and 
conservation. Exp Biol Med 2019; 244: 494-504.

66469 Schmidt EKA, Torres-Espin A, Raposo PJF, Madsen KL, Kigerl KA, Popovich PG, Fenrich

665 KK, Fouad K. Fecal transplant prevents gut dysbiosis and anxiety-like behaviour after spinal $666 \quad$ cord injury in rats. PLoS One 2020; 15: e0226128.

66770 Wei Y, Gong J, Zhu W, Guo D, Gu L, Li N, Li J. Fecal microbiota transplantation restores 668 dysbiosis in patients with methicillin resistant Staphylococcus aureus enterocolitis. BMC $669 \quad$ Infect Dis 2015; 15: 265.

67071 Karlsson FH, Ussery DW, Nielsen J, Nookaew I. A closer look at bacteroides: phylogenetic 671 relationship and genomic implications of a life in the human gut. Microb Ecol 2011; 61:

$672 \quad 473-485$.

67372 Bornbusch SL, Greene LK, McKenney EA, Volkoff SJ, Midani FS, Joseph G, Gerhard WA, 674 Iloghalu U, Granek J, Gunsch CK. A comparative study of gut microbiomes in captive 675 nocturnal strepsirrhines. Am J Primatol 2019; 81: e22986.

67673 Greene LK, Clayton JB, Rothman RS, Semel BP, Semel MA, Gillespie TR, Wright PC, 677 Drea CM. Local habitat, not phylogenetic relatedness, predicts gut microbiome structure 678 within frugivorous and folivorous lemur lineages. .

67974 Macrina FL, Mays TD, Smith CJ, Welch RA. Non-plasmid associated transfer of antibiotic $680 \quad$ resistance in Bacteroides. J Antimicrob Chemother 1981; 8: 77-86.

68175 Whittle G, Shoemaker NB, Salyers AA. The role of Bacteroides conjugative transposons in 682 the dissemination of antibiotic resistance genes. Cell Mol Life Sci C 2002; 59: 2044-2054.

68376 Privitera G, Dublanchet A, Sebald M. Transfer of multiple antibiotic resistance between $684 \quad$ subspecies of Bacteroides fragilis. J Infect Dis 1979; 139: 97-101.

68577 Shoemaker NB, Vlamakis H, Hayes K, Salyers AA. Evidence for extensive resistance gene 
transfer among Bacteroides spp. and among Bacteroides and other genera in the human colon. Appl Environ Microbiol 2001; 67: 561-568.

78 Wexler HM. Bacteroides: the good, the bad, and the nitty-gritty. Clin Microbiol Rev 2007; 20: $593-621$.

79 Veloo ACM, Baas WH, Haan FJ, Coco J, Rossen JW. Prevalence of antimicrobial resistance genes in Bacteroides spp. and Prevotella spp. Dutch clinical isolates. Clin Microbiol Infect 2019; 25: 1156-e9.

80 Stiefel U, Tima MA, Nerandzic MM. Metallo- $\beta$-lactamase-producing Bacteroides species can shield other members of the gut microbiota from antibiotics. Antimicrob Agents Chemother 2015; 59: 650-653. WP, Tiedje JM et al. Choosing your battles: which resistance genes warrant global action? BioRxiv 2019; : 784322. GE, de Geus EJC et al. Cohabitation is associated with a greater resemblance in gut microbiota which can impact cardiometabolic and inflammatory risk. BMC Microbiol 2019; 19: $1-10$. Knights D, Clemente JC, Nakielny S et al. Cohabiting family members share microbiota with one another and with their dogs. Elife 2013; 2: e00458. 34. 
resistance genes in bacteria. Br J Pharmacol 2008; 153.

71086 Ventola CL. The antibiotic resistance crisis: part 2: management strategies and new agents.

$711 \quad$ Pharm Ther 2015; 40: 344.

71287 Van Puyvelde S, Deborggraeve S, Jacobs J. Why the antibiotic resistance crisis requires a

713 One Health approach. Lancet Infect Dis 2018; 18: 132-134.

71488 Bengtsson B, Greko C. Antibiotic resistance — consequences for animal health, welfare, and 715 food production. Ups J Med Sci 2014; 119: 96-102.

71689 Cabello FC. Heavy use of prophylactic antibiotics in aquaculture: a growing problem for

717 human and animal health and for the environment. Environ Microbiol 2006; 8: 1137-1144.

71890 Perry MR, McClean D, Simonet C, Woolhouse M, McNally L. Focusing on resistance to

719 front-line drugs is the most effective way to combat the antimicrobial resistance crisis.

$720 \quad$ bioRxiv 2018; : 498329.

72191 Austvoll CT, Gallo V, Montag D. Health impact of the Anthropocene: the complex

722 relationship between gut microbiota, epigenetics, and human health, using obesity as an

723 example. Glob Heal Epidemiol Genomics 2020; 5.

72492 Gillings MR, Paulsen IT. Microbiology of the Anthropocene. Anthropocene 2014; 5: 1-8.

72593 Stearns SC. Evolutionary medicine: its scope, interest and potential. Proc R Soc B Biol Sci

726 2012; 279: 4305-4321.

72794 Trevathan WR. Evolutionary medicine. Annu Rev Anthropol 2007; 36.

72895 Bornbusch SL, Grebe NM, Lunn S, Southworth CA, Dimac-Stohl K, Drea C. Stable and 729 transient structural variation in lemur vaginal, labial and axillary microbiomes: patterns by $730 \quad$ species, body site, ovarian hormones and forest access. FEMS Microbiol Ecol 2020; 96 : 731 fiaa090. 
73296 Hernando-Amado S, Coque TM, Baquero F, Martínez JL. Defining and combating

733 antibiotic resistance from One Health and Global Health perspectives. Nat Microbiol 2019;

734 4: 1432-1442.

73597 Trinh P, Zaneveld JR, Safranek S, Rabinowitz PM. One health relationships between

736 human, animal, and environmental microbiomes: a mini-review. Front public Heal 2018; 6:

$737 \quad 235$.

73898 Starling AP, Charpentier MJE, Fitzpatrick C, Scordato ES, Drea CM. Seasonality, sociality, 739 and reproduction: long-term stressors of ring-tailed lemurs (Lemur catta). Horm Behav 2010; 57: 76-85.

74199 Drea CM. Sex and seasonal differences in aggression and steroid secretion in Lemur catta:

742 are socially dominant females hormonally 'masculinized'? Horm Behav 2007; 51: 555-567.

743100 Charles-Smith LE, Cowen P, Schopler R. Environmental and physiological factors

744 contributing to outbreaks of Cryptosporidium in Coquerel's Sifaka (Propithecus coquereli)

745 at the Duke Lemur Center: 1999-2007. J Zoo Wildl Med 2010; 41: 438-444.

746101 da Silva AJ, Cacciò S, Williams C, Won KY, Nace EK, Whittier C, Pieniazek NJ, Eberhard

747 ML. Molecular and morphologic characterization of a Cryptosporidium genotype identified

$748 \quad$ in lemurs. Vet Parasitol 2003; 111: 297-307.

749102 Caporaso JG, Lauber CL, Walters WA, Berg-Lyons D, Huntley J, Fierer N, Owens SM,

750 Betley J, Fraser L, Bauer M et al. Ultra-high-throughput microbial community analysis on

751 the Illumina HiSeq and MiSeq platforms. ISME J 2012; 6: 1621.

752103 Hall M, Beiko RG. 16S rRNA gene analysis with QIIME2. In: Microbiome Analysis.

$753 \quad$ Springer, 2018, pp 113-129.

754104 Callahan BJ, McMurdie PJ, Rosen MJ, Han AW, Johnson AJA, Holmes SP. DADA2: high- 
resolution sample inference from Illumina amplicon data. Nat Methods 2016; 13: 581.

756

757

758

759

760

761

762

763

764

765

766

767

768

769

770

771

772

773

774

775

776

777

105 Quast C, Pruesse E, Yilmaz P, Gerken J, Schweer T, Yarza P, Peplies J, Glöckner FO. The SILVA ribosomal RNA gene database project: improved data processing and web-based tools. Nucleic Acids Res 2012; 41: D590-D596.

106 Yarza P, Yilmaz P, Pruesse E, Glöckner FO, Ludwig W, Schleifer K-H, Whitman WB, Euzéby J, Amann R, Rosselló-Móra R. Uniting the classification of cultured and uncultured bacteria and archaea using 16S rRNA gene sequences. Nat Rev Microbiol 2014; 12: 635.

107 Hasan NA, Young BA, Minard-Smith AT, Saeed K, Li H, Heizer EM, McMillan NJ, Isom R, Abdullah AS, Bornman DM et al. Microbial community profiling of human saliva using shotgun metagenomic sequencing. PLoS One 2014; 9: e97699.

108 Yan Q, Wi YM, Thoendel MJ, Raval YS, Greenwood-Quaintance KE, Abdel MP, Jeraldo PR, Chia N, Patel R et al. Evaluation of the CosmosID bioinformatics platform for prosthetic joint-associated sonicate fluid shotgun metagenomic data analysis. J Clin Microbiol 2019; 57.

109 Chekabab SM, Lawrence JR, Alvarado A, Predicala B, Korber DR. A health metadatabased management approach for comparative analysis of high-throughput genetic sequences for quantifying antimicrobial resistance reduction in Canadian hog barns. Comput Struct Biotechnol J 2020.

110 Feehan A, Garcia-Diaz J. Bacterial, gut microbiome-modifying therapies to defend against multidrug resistant organisms. Microorganisms 2020; 8: 166.

111 Pedersen EJ, Miller DL, Simpson GL, Ross N. Hierarchical generalized additive models in ecology: an introduction with mgcv. PeerJ 2019; 7: e6876.

112 Gloor GB, Macklaim JM, Fernandes AD. Displaying variation in large datasets: plotting a 
visual summary of effect sizes. J Comput Graph Stat 2016; 25: 971-979.

779113 Fernandes AD, Reid JN, Macklaim JM, McMurrough TA, Edgell DR, Gloor GB. Unifying

780 the analysis of high-throughput sequencing datasets: characterizing RNA-seq, 16S rRNA

781 gene sequencing and selective growth experiments by compositional data analysis.

$782 \quad$ Microbiome 2014; 2: 15.

783114 Gloor GB, Macklaim JM, Pawlowsky-Glahn V, Egozcue JJ. Microbiome datasets are

784 compositional: and this is not optional. Front Microbiol 2017; 8: 2224.

785

786 